\title{
Nanoscale Photocurrent Microscopy for Thin Film Solar Cells Using Focused Electron Beam and Near-Field Optical Excitations
}

\author{
Heayoung P. Yoon ${ }^{1,2}$, Yohan Yoon ${ }^{1,2}$, Paul M. Haney ${ }^{1}$, Sangmin An ${ }^{1,2}$, Joshua Schumacher ${ }^{1}$, Kerry \\ Siebein $^{1}$, Alline Myers ${ }^{1}$, and Nikolai B. Zhitenev ${ }^{1}$ \\ ${ }^{1}$ National Institute of Standards and Technology, Gaithersburg, MD, 20889, USA, \\ ${ }^{2}$ Maryland NanoCenter, University of Maryland, College Park, MD 20742, USA
}

Characterization techniques based on scanning probe microscopy are increasingly used for investigating microstructure, compositions, and optoelectronic properties of photovoltaic devices. In photocurrent microscopy, excess carriers are generated by an injected beam of photons, which are collected by a Schottky contact or a $p$ - $n$ junction. The value of measured photocurrent corresponds to the local efficiency of carrier collection, which is determined by the local built-in potential and applied electric field, as well as the carrier recombination rate. This direct imaging technique has been frequently used for characterizing bulk properties of semiconductor devices (e.g., defects, diffusion lengths).

Thin film solar cells (e.g., $\left.\mathrm{CdTe}, \mathrm{Cu}\left(\mathrm{In}_{\mathrm{x}} \mathrm{Ga}_{1-\mathrm{x}}\right) \mathrm{Se}_{2}\right)$ are comprised of grains with sizes on the order of 1 $\mu \mathrm{m}$ (Figure 1a). Previous work suggested that their inhomogeneous properties significantly impact the overall photovoltaic performance [1]. However, photocarrier dynamics in the microstructures (e.g., grain bulk, grain boundary, metallurgical junction) are currently not well understood. In this work, we measure local quantum efficiency of individual microstructures using near-field scanning optical microscopy (NSOM). Furthermore, we perform electron-beam induced current (EBIC) measurements that provide higher spatial resolution $(<20 \mathrm{~nm})$. EBIC allows systematic controls of the carrier generation and of the size of interaction volume (tens of $\mathrm{nm}$ to a few um), well-suited for quantitative study.

An Ohmic metal contact was formed to $p$-CdTe and $n-\mathrm{CdS} / \mathrm{SnO}_{2}$ layers [2]. In order to measure the local response throughout the entire $p-n$ junction, cross-sections of the device were prepared using focused ion beam (FIB) processes. For NSOM, we used a tapered optical fiber probe (200 nm diameter; Figure 1b) mounted on a tuning fork. Figures 1 (c) and (d) show the photocurrent maps at illumination wavelengths of $405 \mathrm{~nm}$ and $630 \mathrm{~nm}$, respectively. The spatial resolution decreases at the longer wavelengths, as expected, due to the increase of the absorption depth. The bright contrast at the grain boundaries indicates high carrier collection efficiency, as proposed in previous works $[2,3]$. We fit the line-scan collected near a center of a single grain under $635 \mathrm{~nm}$ illumination to estimate the minority carrier diffusion length $\left(L_{n}\right)$ of $\approx 0.7 \mu \mathrm{m}$ (Figure $1 \mathrm{~b}$ )

Figure 2 shows a series of cross-sectional EBIC images obtained at different acceleration voltages. The strong bright contrast at grain boundaries reflects the high carrier collections as seen in NSOM (Figure 1 c). Qualitatively, the $5 \mathrm{keV}$ and $10 \mathrm{keV}$ EBIC images are similar to that of $405 \mathrm{~nm}$ and $603 \mathrm{~nm}$ NSOM maps, respectively. This correspondence indicates that the calculated photon absorption and electron penetration depths are similar in magnitude. To extract material parameters, we performed least-squares fitting of the individual EBIC line scans using an analytical model that deconvolutes the collection efficiency and the generation profile [4]. Figure 2 (d) plots EBIC line scans overlaid with the model fit for a large, single grain. The extracted minority carrier diffusion lengths at different acceleration voltages were in the range of $0.6 \mu \mathrm{m}$ to $0.8 \mu \mathrm{m}$, showing good agreement with the estimate from NSOM 
(Figure 2 b). Figure 2 also shows that the maximum collection efficiency is below 1 . We attribute this reduced efficiency to strong surface recombination. Development of an analytical model that accounts for surface recombination in the depletion region and screening of built-in fields from carrier accumulation is in progress, and it will provide more information on the local properties (e.g., depletion width, carrier mobility and lifetime).

In summary, we present local photocurrent microscopies of a cross-sectional CdTe solar cell using excitations by near-field optical illumination and by focused electron beam irradiation. The spatially and spectrally resolved efficiency maps confirm a higher carrier collection at grain boundaries. We estimate the minority carrier diffusion lengths of individual grains measured away from grain boundaries from NSOM and EBIC data and demonstrate an excellent agreement between the techniques.

[1] S. Kumar and K. Rao, Energy Environ. Sci., 7 (2014), p.45

[2] H. P. Yoon et al., Solar Energy Mater. Solar Cells 117 (2013)

[3] J. D. Poplawsky et al., Adv. Energy Mater., 1400454 (2014), p. 499.

[4] P. M. Haney et al., arXiv:1410.4435v1, 2014.
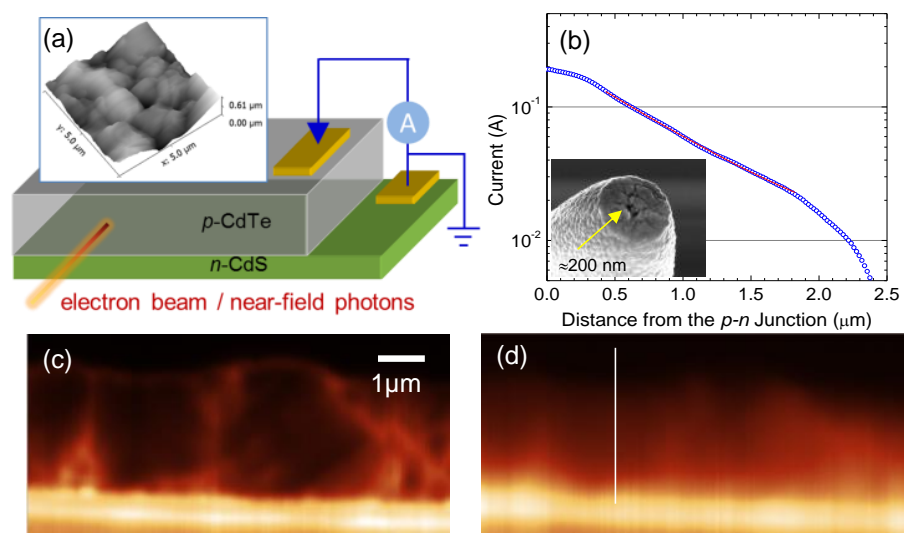

Figure 1. (a) Schematic of photocurrent microscopy. The inset shows a topography of a CdTe solar cell. (b) An NSOM line-scan profile at $635 \mathrm{~nm}$ illumination (white line in d). NSOM image at 405 $\mathrm{nm}(\mathrm{c})$ and $635 \mathrm{~nm}(\mathrm{~d})$. The estimated photon absorption depth is $100 \mathrm{~nm}$ and $400 \mathrm{~nm}$, respectively.
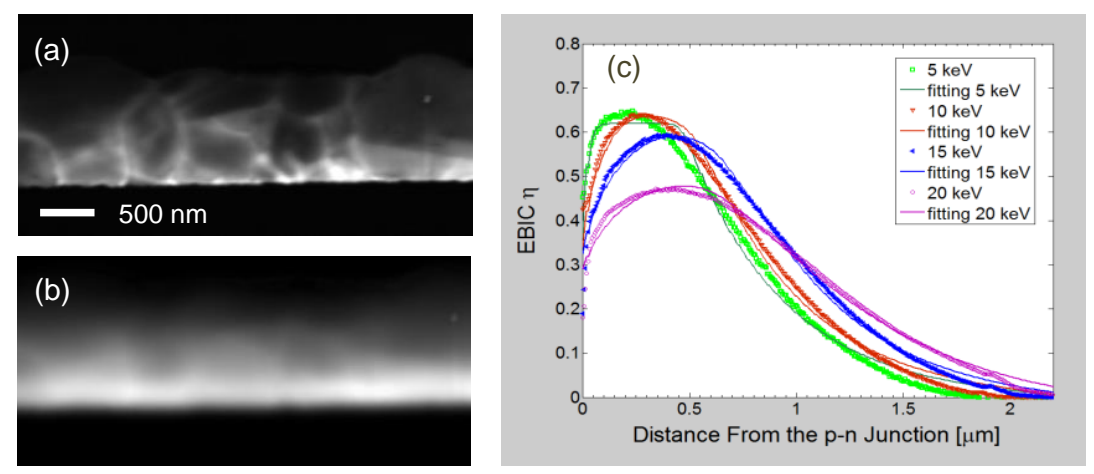

Figure 2. Cross-sectional EBIC images at $3 \mathrm{keV}$ (a) and $10 \mathrm{keV}$ (b). The calculated penetration depth in CdTe is $40 \mathrm{~nm}$ and $310 \mathrm{~nm}$ respectively. (c) Measured EBIC linescans (solid lines) obtained for a large, single grain, overlaid with the model fit (dot lines) 